\title{
Current distribution control of dual directly driven wheel motors for electric vehicles
}

\author{
Yee-Pien Yang*, Chun-Pin Lo \\ Department of Mechanical Engineering, National Taiwan University, No. 1, Roosevelt Road, Sec. 4, Taipei, Taiwan 106, ROC
}

\section{A R T I C L E I N F O}

Article history:

Received 2 October 2006

Accepted 25 February 2008

\section{Keywords:}

Current distribution control

Directly driven wheel motor

Electric vehicle

\begin{abstract}
A B S T R A C T
This paper proposes a current distribution control for dual directly driven wheel motors for electric vehicles. The objective is to maintain two driving wheels at a synchronous speed in order to keep the vehicle straight, or at differential speeds when cornering, even when they incur uneven load disturbance or parameter changes. The proposed control scheme employs a load disturbance observer, a model following controller, and a velocity command compensator to determine the proper amount of current supplied to each driving wheel. The vehicle dynamics and control strategy were modeled and the control performance was simulated numerically. Experiments were performed in a hardware-inthe-loop configuration with a dedicated wheel motor on a dynamometer and a virtual one on a field programmable gate array chip where the current distribution control was implemented. The resulting control performance verified the stability and robustness of the system in terms of its insensitivity to parameter variations and its rejection of external disturbances.
\end{abstract}

(c) 2008 Elsevier Ltd. All rights reserved.

\section{Introduction}

Electric vehicles (EVs) are developing fast during this decade due to drastic issues on the protection of environment and the shortage of energy sources. While commercial hybrid cars have been rapidly exposed on the market, fuel-cell-powered vehicles are also announced to appear in 5-10 years. Researches on the power propulsion system of EVs have drawn significant attention in the automobile industry and among academics. EVs can be classified into various categories according to their configurations, functions or power sources. Pure EVs do not use petroleum, while hybrid cars take advantages of energy management between gas and electricity (Poursamad and Montazeri, 2007). Indirectly driven EVs are powered by electric motors through transmission and differential gears, while directly driven vehicles are propelled by in-wheel or, simply, wheel motors (Chen \& Chau, 2001).

The basic vehicle configuration of this research has two directly driven wheel motors installed and operated inside the driving wheels on a pure EV. These wheel motors can be controlled independently and have so quick and accurate response to the command that the vehicle chassis control or motion control becomes more stable and robust, compared to indirectly driven EVs. Like most research on the torque distribution control of

\footnotetext{
* Corresponding author. Tel.: +8862 3366 2682; fax: +886223631755.

E-mail address: ypyang@ntu.edu.tw (Y.-P. Yang).
}

wheel motors, He, Hori, Kamachi, Walters, and Yoshida (2005) proposed a dynamic optimal tractive force distribution control for an EV driven by four wheel motors, thereby improving vehicle handling and stability. The researchers assumed that wheel motors were all identical with the same torque constant, neglecting motor dynamics-the output torque was simply proportional to the input current with a prescribed torque constant.

Under this assumption, Hori, Toyoda, and Tsuruoka (1998) proposed a model following control and an optimal slip ratio control to adjust tractive torque when the motor speed is suddenly increased by tire slip, under the assumption of equal torque and friction forces on the right and left tires. The model following control and the slip ratio control was effectively implemented on their experimental EV, the UOT March II, with four in-wheel motors (Hori, 2004). Mutoh and Higashikubo (2002) introduced a control structure to distribute torque to the front and rear wheels, which were driven independently and indirectly by a synchronous motor and an induction motor. A similar method known as direct yaw moment control was proposed by Sakai, Sado, and Hori (2002), where the vehicle's lateral motion was controlled by a yaw moment generated by the torque difference between wheel motors. Hallowell and Ray (2003) implemented an all-wheel-driven 1/8 scale vehicle model, with a wheel slip controller for each wheel and a central torque distribution controller to enhance lateral stability and follow the driver's intended trajectory. Pusca, Ait-Amirat, Berthon, and Kauffmann (2002) modeled and simulated a traction control 
algorithm for an EV with four separate drives to ensure lateral dynamic stability.

No previous researchers have taken into consideration the differences in motor dynamics among wheel motors, whose internal uncertainties, such as variation in motor parameters or fault motor components, may cause risky driving due to instability. When the dynamics of a wheel motor are considered, the input current produces output torque according to the variation of motor parameters, such as winding resistance, inductance, and viscous or dry friction, due to the change in temperature, manufacturing tolerance or aging effects. The same amount of current may produce different torque outputs from different wheel motors given the same road condition, thereby yielding different velocities and causing undesirable sideslip. It is therefore quite necessary to produce the desired torque for each wheel by properly distributing the current, subject to the variation of motor parameters as well as to external disturbances. In short, current distribution control is equivalent to torque distribution control when motor dynamics are incorporated in the control strategy.

This paper constructs an internal controller to distribute current to the driving wheels of EVs. Since wheel motors may be fabricated of different qualities, and age at different rates, the same current may not produce the same torque output. An uneven torque distribution to driving wheels will cause undesired sideslip in the cruising and cornering modes. The proposed internal controller serves to distribute the current, instead of torque, to the driving wheels, given different dynamics and uncertain loads from tires, thereby enhancing the robustness and stability of the system.

\section{Wheel motor and its dynamics}

The custom-designed wheel motor is a directly driven disc-type axial-flux brushless dc motor. The rotor is embedded with 18 fanshaped magnets and sandwiched by two plates of stator. Each stator is toroidally wound with a strip of continuous steel to form 24 teeth. The coils are independently wired on stator poles and grouped into four phases, each of which is bound with six parallelconnected windings. The same phase on the left and right plates can be connected in two modes: serial and parallel for a singlephase model. The parallel mode is suitable for high-speed operation at higher phase voltage, while the serial mode for lowspeed operation operates at a lower phase voltage. Its maximum torque is $64 \mathrm{~N} \mathrm{~m}$, the maximum speed is $950 \mathrm{rpm}$, and the rated power is $1.85 \mathrm{~kW}$ at $340 \mathrm{rpm}$. The tire is mounted on the outer case that rotates with the rotor of the wheel motor. Its exploded graph and stator phase windings are drawn in Fig. 1, and its optimal design and control are addressed in Yang, Luh, and Cheung (2004) and in Yang, Wang, Wu, and Luh (2004). Fig. 2 illustrates the installation of two rear driving wheel motors on an EV.
The electrical and mechanical equations of the wheel motor are derived under the assumptions that (i) each wheel motor is an axial-flux dc brushless motor, (ii) the motor is operated in the linear range of the $B-H$ curve of the magnetic material, and (iii) the flux flows straightly across the air-gaps between the stator and rotor, ignoring the fringing flux (Hanselman, 2003). The equations of motion of the vehicle driven by two rear wheels are written with other assumptions: (i) the tractive effort of two front tires is zero, (ii) the wheel mass is negligibly small compared with the vehicle mass, and (iii) the tire, yawing, pitching and rolling dynamics are not considered (Wong, 1978). Therefore, the dynamic equations of each rational wheel motor can be expressed as

$T=4 k_{\mathrm{t}} I$,

$V=I R+L \frac{\mathrm{d} I}{\mathrm{~d} t}+K_{\mathrm{e}} \omega$

$T=J \dot{\omega}+D \omega+T_{\mathrm{L}}$,

where $T$ is the produced torque, $I$ is the input current, $K_{\mathrm{t}}$ is the torque constant as a function of motor parameters, the multiplier 4 accounts for four phases, $V$ is the phase voltage, $R$ is the phase resistance, $L$ is the phase inductance, $K_{\mathrm{e}}$ is the back emf constant, $\omega$ is the rotational wheel speed, $J$ and $D$ are the wheel inertia and damping coefficient, respectively. The external load on tire $T_{\mathrm{L}}$ can simply account for the tractive torque $f_{i} r$ produced by the friction between ground and tire on each driving wheel $i$, where $r$ is the tire radius. It is assumed that the wheels roll without slipping, thus $v_{i}=r \omega_{i}$; the friction $f_{i}$, which is less than the maximum static friction, becomes the tractive effort on the vehicle. When the phase inductance is neglected in the steady state, the combination of (1) through (3) yields

$\dot{v}_{i}=-\left(\frac{D_{i} R_{i}+4 K_{\mathrm{t} i} K_{\mathrm{e} i}}{J_{i} R_{i}}\right) v_{i}+\left(\frac{4 K_{\mathrm{t} i} V_{i}-f_{i} r R_{i}}{J_{i} R_{i}}\right) r$,

where $i=a$ and $b$, denoting rear wheels A and B.
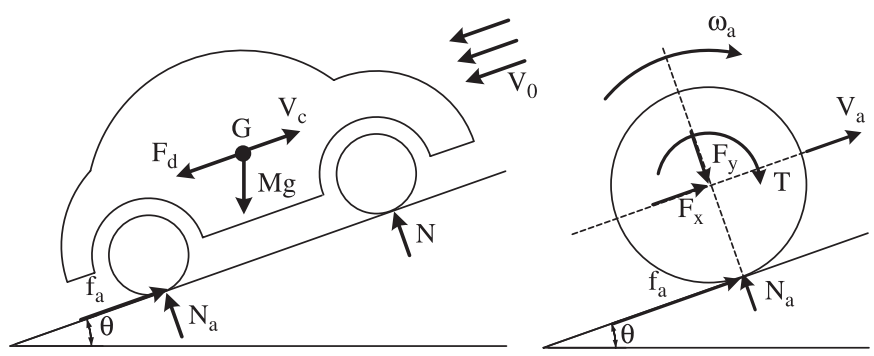

Fig. 2. (a) Free body diagram of driving wheel $A$ and (b) longitudinal vehicle configuration.
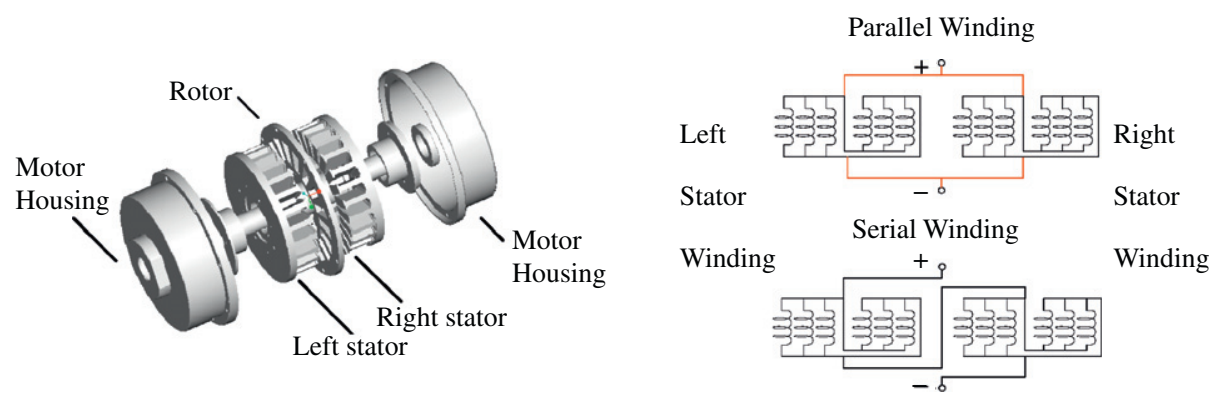

Fig. 1. Exploded graph of axial-flux dc brushless wheel motor and its stator phase windings. 
Without considering tire, yawing, pitching and rolling dynamics, the equation of motion of the vehicle, according to Fig. 2, along the longitudinal direction is simply

$M \dot{v}_{\mathrm{c}}=\left(f_{\mathrm{a}}+f_{\mathrm{b}}\right)-F_{\mathrm{d}}$,

in which

$F_{\mathrm{d}}=N_{\mathrm{T}} C_{\mathrm{r}}+\frac{1}{2} \rho\left(v_{\mathrm{c}}+v_{0}\right)^{2} A C_{\mathrm{d}}+M g \sin (\theta)$,

where $F_{\mathrm{d}}$ denotes the total drag force on the vehicle, $M$ is the vehicle mass, $N_{\mathrm{T}}$ is the total normal force of the vehicle, $\rho$ is the air density, $A$ is the frontal area of the vehicle, $C_{\mathrm{d}}$ is the air drag coefficient, $v_{0}$ is the absolute velocity of wind, $C_{\mathrm{r}}$ is the rolling resistance coefficient, and $\theta$ is the slope angle of the road. In the ideal situation of rolling without slipping, the vehicle velocity $v_{\mathrm{c}}$ is the same as the longitudinal velocities $v_{\mathrm{a}}$ and $v_{\mathrm{b}}$ of the driving wheels.

\section{Current distribution control}

The dedicated wheel motors are of low speed and high torque, and are driven by an optimal current waveform, which has been proven to be of the same form as the back emf of the wheel motor (Yang, Luh, \& Cheung, 2004; Yang, Wang, Wu, \& Luh, 2004). The driver depresses the accelerator pedal to make a driving command, which is transformed into equally distributed currents so that the left and right wheels gain the same amount of torque to guarantee straight movement of the vehicle. This ideal situation happens when the dynamics and external loads of the two driving motors are exactly the same. Although the slip mechanism is not considered in the internal control loop described in this paper, the two driving wheels are expected to be running at the same velocity in the cruising mode under the uncertainties of motor dynamics and external disturbances. In other words, the proposed controller must be robust and stable, and have quick response. To achieve the objective, a current distribution control scheme is proposed, which consists of a pair of model following controls (MFC) with load disturbance observers, proportional-integral (PI) velocity controllers, and a velocity command compensator (VCC), as shown in Fig. 3.

\subsection{Model following control and load observer}

The driving command from the accelerator pedal is transformed to a current command $I_{\text {com }}$, which is used to determine the velocity command in the successive step of velocity control on the driving wheels. The nominal model of a wheel motor, which is coded with nominal parameters, is responsible for calculating the corresponding velocity $\omega^{*}$ by $(1 / 2) I_{\text {com }}$ from (1) and (3). Concurrently, the real input current $I^{*}$ and output velocity $\omega$ are measured, and the external load $T_{\mathrm{L}}^{\prime}$ is estimated adaptively by a load disturbance observer according to the following equation:

$T_{\mathrm{L}}^{\prime}=K_{\mathrm{t}}^{\prime} I^{*}-J^{\prime} \frac{\mathrm{d} \omega}{\mathrm{d} t}-D^{\prime} \omega$,

where $K_{\mathrm{t}}^{\prime}, J^{\prime}$ and $D^{\prime}$ are the nominal torque constant, inertia and viscous damping coefficients of the wheel motor, respectively. To eliminate the noise due to velocity differentiation, the estimated load is processed through a low-pass filter before being sent to the MFC.

\subsection{Velocity command compensator}

The control objective for the dual wheels is to synchronize their velocities despite differences in motor dynamics and external disturbances. The other crucial point is to guarantee that the summation of the real currents $I_{\mathrm{a}}^{*}$ and $I_{\mathrm{b}}^{*}$ to the driving wheels is equal to the command $I_{\text {com }}$ from the driver. In other words, the "power" that flows into the vehicle is fully "authorized" by the driver, and the vehicle is completely controllable. Notice that the following equations are expressed in the Laplace domain. The velocity compensation is easily derived from (1) and (3) by investigating the deviation of wheel speed with respect to the variance of current:

$\Delta \omega(s)=\frac{4 K_{\mathrm{t}}}{J s+D} \Delta I(s)$.

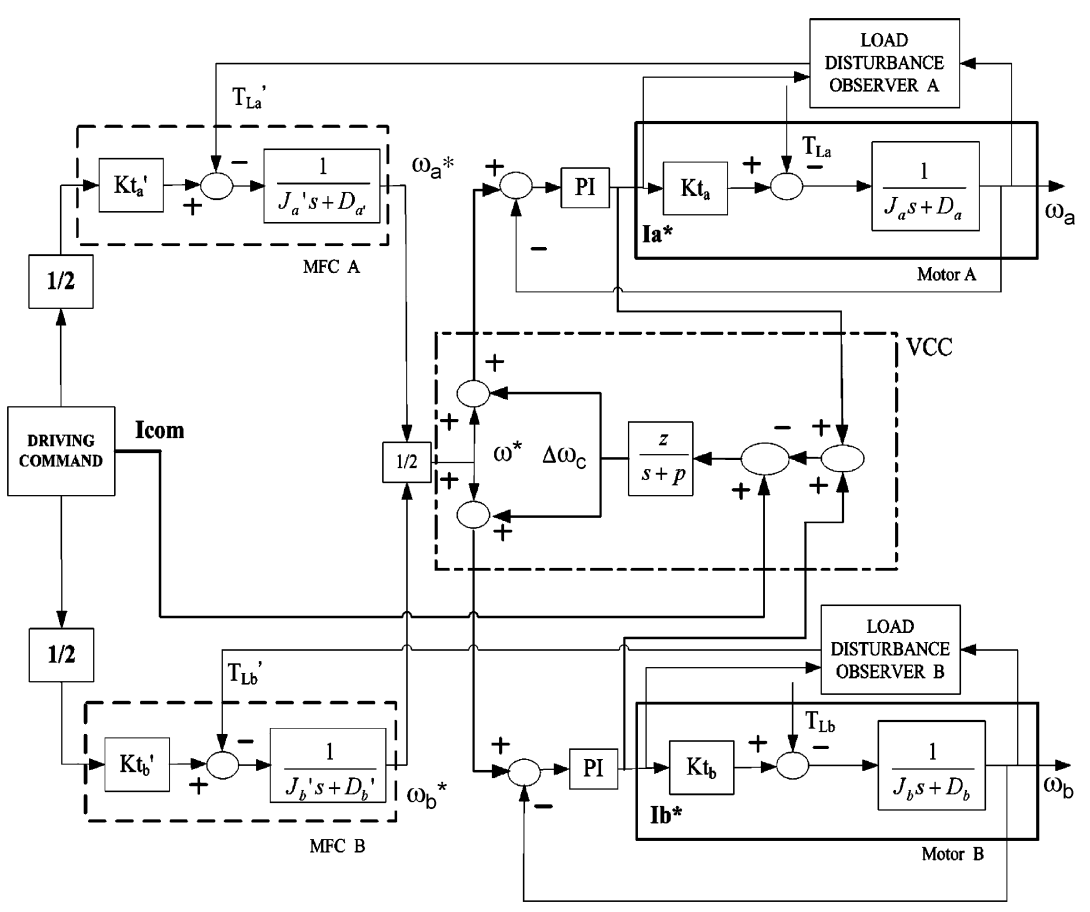

Fig. 3. Current distribution control configuration. 


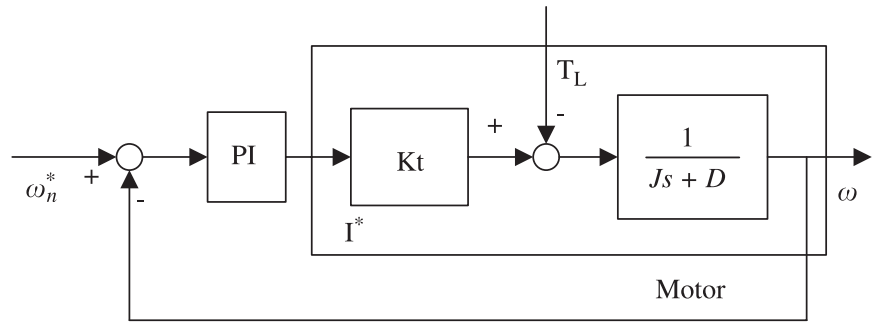

Fig. 4. PI velocity controller.

Therefore, the new velocity command becomes

$\omega_{\mathrm{n}}^{*}=\omega^{*}+\Delta \omega_{\mathrm{c}}$,

where $\omega^{*}$ is generated from the MFC block. Since the left and right wheel motors may have different parameters, the first-order transfer function in (8) is replaced by a properly selected $z /\left(s^{+} p\right)$, so that $\Delta I=I_{\text {com }}-\left(I_{\mathrm{a}}^{*}+I_{\mathrm{b}}^{*}\right)$ approaches zero in the steady state.

\subsection{PI velocity controller}

After determining the new velocity command, the PI velocity controller is employed to calculate the current command to each wheel motor, as shown in Fig. 4. The stability and performance of the control system are dependent on properly tuned proportional and integral gains $K_{\mathrm{p}}$ and $K_{\mathrm{l}}$. Without external disturbances, the transfer function between the wheel speed $\omega$ and $\omega_{\mathrm{n}}^{*}$ is expressed by

$G(s)=\frac{\omega}{\omega_{\mathrm{n}}^{*}}(s)=\frac{\left(K_{\mathrm{p}} s+K_{\mathrm{I}}\right) K_{\mathrm{t}}}{J s^{2}+\left(D+K_{\mathrm{p}} K_{\mathrm{t}}\right) s+K_{\mathrm{I}} K_{\mathrm{t}}}$.

This is required for both the left and right wheels, and is denoted by $G_{\mathrm{a}}(s)$ and $G_{\mathrm{b}}(s)$, respectively.

\section{Stability and robustness analysis}

The stability and robustness of the current distribution controller are investigated on the dual directly driven wheel motors. Although vehicle dynamics, such as yawing, pitching and rolling, are not modeled in this research, all their influence on the wheel motor is lumped into the tractive torque $T_{\mathrm{L}}$ in (3), which is regarded as a load disturbance to the wheel motor in the following analyses. Therefore, the stability and robustness analyses are still effective for more rigorous models of vehicle dynamics.

\subsection{Stability analysis}

The stability of the current distribution controller is investigated by the transfer function between $\left(I_{\mathrm{a}}^{*}+I_{\mathrm{b}}^{*}\right)$ and $I_{\mathrm{com}}$, which is obtained by assembling MFC, VCC, and PI velocity controller as follows:

$$
\begin{aligned}
H_{\mathrm{c}}(s) & \equiv \frac{I_{\mathrm{a}}^{*}+I_{\mathrm{b}}^{*}}{I_{\mathrm{com}}} \\
& =\frac{\frac{1}{4}\left(A_{1}(s)+A_{2}(s)+B_{1}(s)+B_{2}(s)\right)+(M(s)+N(s))}{1+M(s)+N(s)},
\end{aligned}
$$

where

$$
\begin{aligned}
& M(s)+N(s) \equiv \frac{z}{s+p}\left[G_{\mathrm{a}}(s) \frac{J_{\mathrm{a}} s+D_{\mathrm{a}}}{K_{\mathrm{ta}}}+G_{\mathrm{b}}(s) \frac{J_{\mathrm{b}} s+D_{\mathrm{b}}}{K_{\mathrm{tb}}}\right], \\
& A_{1}(s)=G_{\mathrm{a}}(s) \frac{K_{\mathrm{ta}}^{\prime}}{K_{\mathrm{ta}}} \frac{J_{\mathrm{a}} s+D_{\mathrm{a}}}{J_{\mathrm{a}}^{\prime} s+D_{\mathrm{a}}^{\prime}}, \quad A_{2}(s)=G_{\mathrm{a}}(s) \frac{K_{\mathrm{ta}}^{\prime}}{K_{\mathrm{ta}}} \frac{J_{\mathrm{a}} s+D_{\mathrm{a}}}{J_{\mathrm{b}}^{\prime} s+D_{\mathrm{b}}^{\prime}}, \\
& B_{1}(s)=G_{\mathrm{b}}(s) \frac{K_{\mathrm{ta}}^{\prime}}{K_{\mathrm{tb}}} \frac{J_{\mathrm{b}} s+D_{\mathrm{b}}}{J_{\mathrm{b}}^{\prime} s+D_{\mathrm{b}}^{\prime}}, \quad B_{2}(s)=G_{\mathrm{b}}(s) \frac{K_{\mathrm{ta}}^{\prime}}{K_{\mathrm{tb}}} \frac{J_{\mathrm{b}} s+D_{\mathrm{b}}}{J_{\mathrm{a}}^{\prime} s+D_{\mathrm{a}}^{\prime}} .
\end{aligned}
$$

Note that $M(s)$ represents the transfer function between current input $I_{\mathrm{a}}^{*}$ and current error $\Delta I$, while $N(s)$ is between $I_{\mathrm{b}}^{*}$ and $\Delta I$. Without detailed proof, the system $H_{\mathrm{c}}(s)$ is stable if $A_{1}(s), A_{2}(s)$, $B_{1}(s), B_{2}(s)$, and $R(s)$ are stable, as long as the pole location $p$ is chosen in the stable region. If the driving command $I_{\text {com }}$ is a step function, the steady-state error becomes

$e_{\mathrm{ss}}=\lim _{s \rightarrow 0} s\left[H_{\mathrm{c}}(s)-1\right] \frac{I_{\mathrm{com}}}{s}=\frac{\left(E_{\mathrm{p}}-1\right) p}{p+z E_{\mathrm{c}}} I_{\mathrm{com}}$,

where

$E_{\mathrm{p}}=\lim _{s \rightarrow 0} \frac{1}{4}\left(A_{1}(s)+A_{2}(s)+B_{1}(s)+B_{2}(s)\right)$

and

$E_{\mathrm{c}}=\lim _{s \rightarrow 0} R(s)=\frac{D_{\mathrm{a}}}{K_{\mathrm{ta}}}+\frac{D_{\mathrm{b}}}{K_{\mathrm{tb}}}$.

Since $E_{\mathrm{p}}$ and $E_{\mathrm{c}}$ are constants, the gain $z$ can be chosen to be large enough to make $e_{\mathrm{ss}}$ approach zero as time goes on. If $p$ is chosen at zero, the VCC reduces to an integral controller, and the resultant current $\left(I_{\mathrm{a}}^{*}+I_{\mathrm{b}}^{*}\right)$ follows the command $I_{\text {com }}$ exactly with zero steady-state error.

\subsection{Robustness analysis}

\subsubsection{PI velocity controller to load disturbance}

From Fig. 4, the transfer function between motor speed and load disturbance is

$G_{\mathrm{L}}(s) \equiv \frac{\omega}{T_{\mathrm{L}}}(s)=\frac{s}{J s^{2}+\left(D+K_{\mathrm{t}} K_{\mathrm{p}}\right) s+K_{\mathrm{t}} K_{\mathrm{I}}}$.

Since the spectrum of external disturbances from a rough road usually has limited bandwidth, the steady-state error tends to be zero. In the special case of a constant disturbance $T_{\mathrm{L}}$, the steadystate error is zero as follows:

$e_{\mathrm{ss}}=\lim _{s \rightarrow 0} s \frac{s}{J s^{2}+\left(D+K_{\mathrm{t}} K_{\mathrm{P}}\right) s+K_{\mathrm{t}} K_{\mathrm{I}}} \frac{T_{\mathrm{L}}}{s}=0$.

\subsubsection{Current distribution controller to system parameters}

Let $H(s)$ be $\left(A_{1}(s)+A_{2}(s)+B_{1}(s)+B_{2}(s)\right) / 4$ that contains all motor parameters, and $C(s)$ be $M(s)+N(s)$ that describes the VCC and PI dynamics of the current distribution controller. Eq. (11) becomes

$H_{\mathrm{c}}(s)=\frac{H(s)+C(s)}{1+C(s)}$.

The sensitivity of $H_{\mathrm{c}}(s)$ with respect to $H(s)$ can be derived as

$S_{H}^{H_{c}}=\frac{\partial H_{\mathrm{c}}}{\partial H} \frac{H}{H_{\mathrm{c}}}=\frac{H(s)}{H(s)+C(s)}$,

which approaches zero if $|C(s)|$ is large enough. This explains that the current distribution control system is not sensitive, or robust, to large variations in system parameters in terms of motor resistance, inductance and damping coefficient. Without VCC or when $C(s)=0$, however, the sensitivity function with respect to $H(s)$ reduces to 1 , revealing a strong sensitivity of the current distribution control to the system parameter change.

\section{Simulation and experiments}

\subsection{Simulation}

The simulation models of the current distribution control and motor dynamics are created with Matlab/Simulink software. The driving wheels are installed with four-phase axial-flux dc 
brushless motors. They are propelled by an optimal current wave, proportional to the back emf of the wheel motor. The parameters of wheel motor A are estimated by step response experiments using a dynamometer, while wheel motor B is a virtual one with assigned parameters. The parameters of VCC and the PI controller are also assigned and listed in Table 1. It is noted that each motor has different dynamic parameters, but the same coefficients are used for their PI velocity controllers.

Table 1

System parameters in simulation

\begin{tabular}{|c|c|c|c|c|c|c|c|c|}
\hline Motor & $J$ & $J^{\prime}$ & $D$ & $D^{\prime}$ & $K_{\mathrm{T}}$ & $K_{\mathrm{T}}^{\prime}$ & $K_{\mathrm{E}}$ & $K_{\mathrm{E}}^{\prime}$ \\
\hline $\begin{array}{l}\text { A } \\
\text { B } \\
\text { Unit }\end{array}$ & $\begin{array}{l}.275 \\
.290 \\
\mathrm{~kg} \mathrm{~m}^{2}\end{array}$ & $\begin{array}{l}.275 \\
.290\end{array}$ & $\begin{array}{l}.22 \\
.10 \\
\mathrm{~N} \mathrm{~m} /(\mathrm{rad} / \mathrm{s})\end{array}$ & $\begin{array}{l}.22 \\
.10\end{array}$ & $\begin{array}{l}.356 \\
.350 \\
\mathrm{~N} \text { m/A }\end{array}$ & $\begin{array}{l}.356 \\
.350\end{array}$ & $\begin{array}{l}.711 \\
.700 \\
\mathrm{~V} /(\mathrm{rad} / \mathrm{s})\end{array}$ & $\begin{array}{l}.711 \\
.700\end{array}$ \\
\hline \multirow[t]{2}{*}{ VCC } & $z$ & & $p$ & & PI & & $20(1+1 / s)$ & \\
\hline & 20 & & 0 & & & & & \\
\hline
\end{tabular}

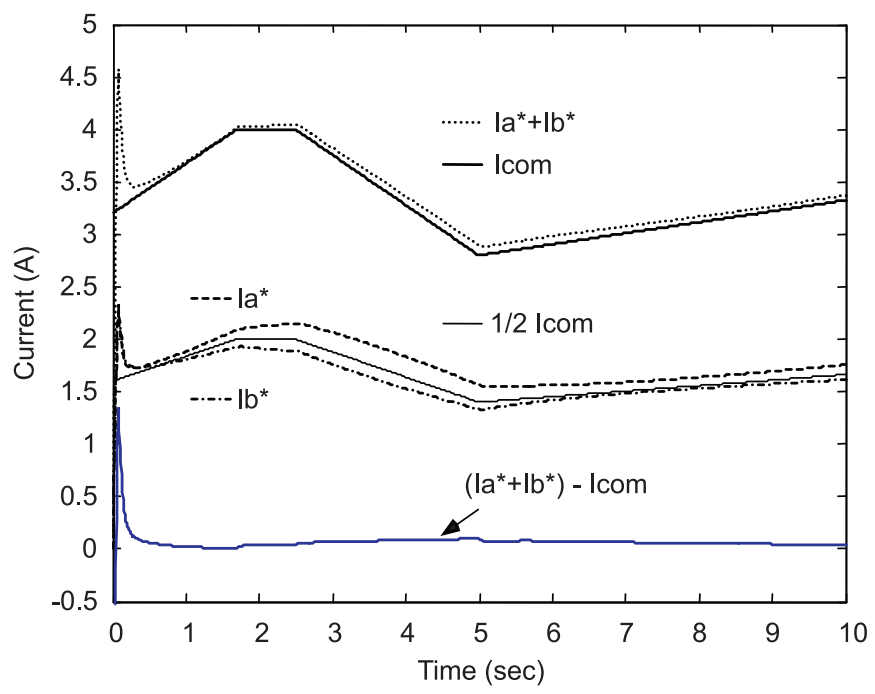

Fig. 5. Current distribution control without VCC (load: $19.62 \mathrm{Nm}$ ).

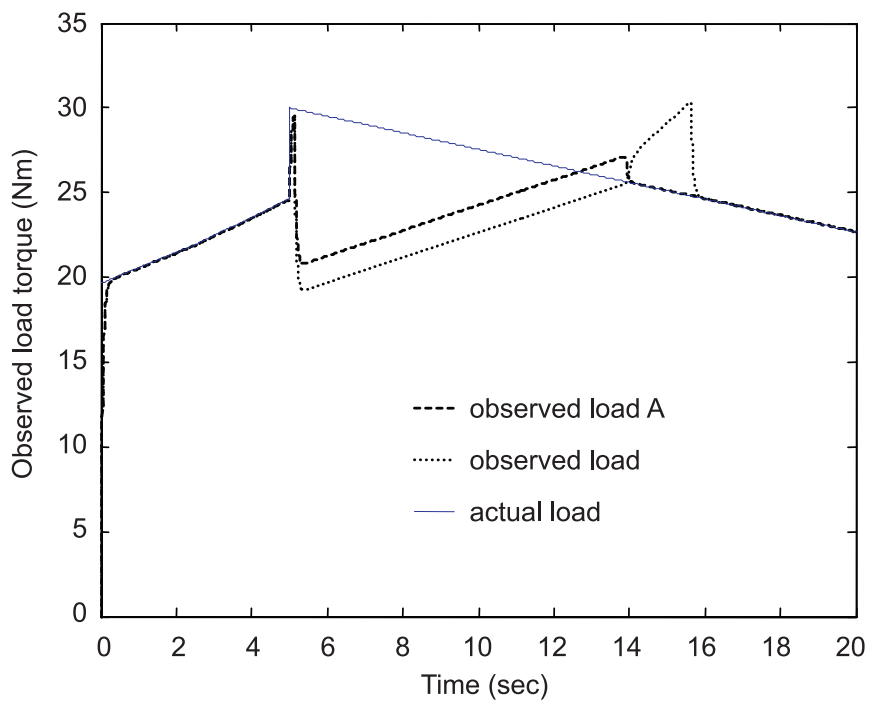

Fig. 6. Actual and estimated load curves.

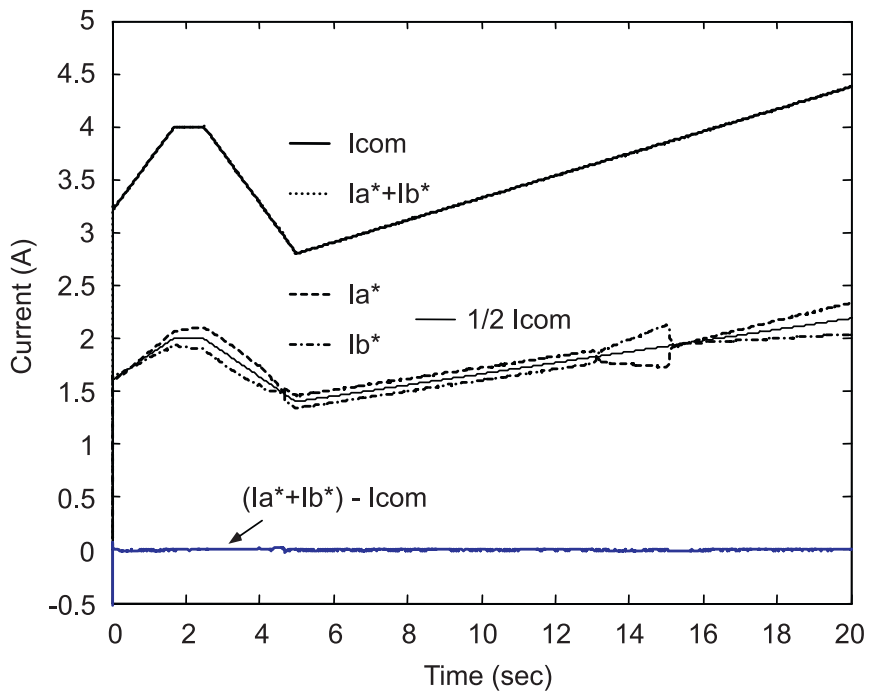

Fig. 7. Current distribution control under load disturbance.

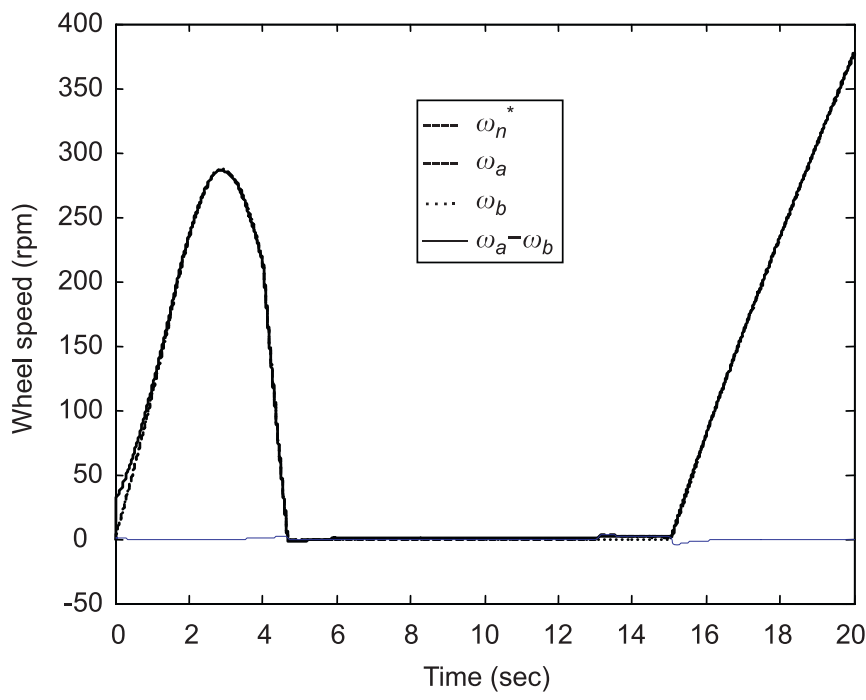

Fig. 8. Dual wheel speeds and their difference with velocity command compensator.

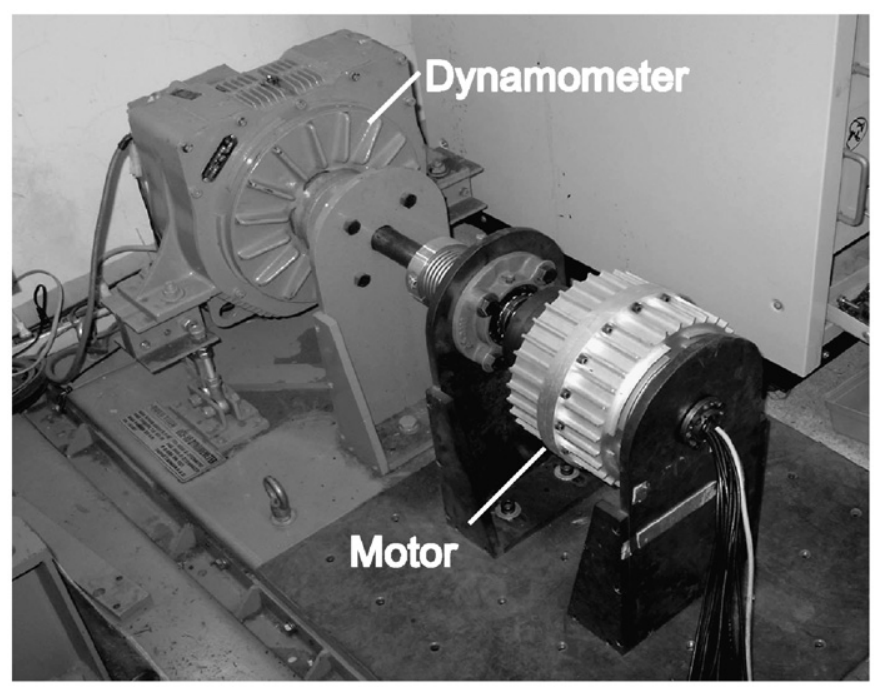

Fig. 9. The wheel motor and dynamometer. 
The driving command $I_{\text {com }}$ is a time function that is composed of accelerating, cruising, and decelerating phases, as shown in Fig. 5. Without the velocity command compensator, the inaccuracy in load estimation and the differences between the dynamics of the two driving motors would yield an undesirable velocity command. The total current $\left(I_{\mathrm{a}}^{*}+I_{\mathrm{b}}^{*}\right)$ fed to motors could become larger than the command $I_{\text {com }}$, putting the driver into a dangerous situation when the car rushes forward out of control.

Fig. 6 shows a case where the external loads vary abruptly, and the load observers are unable to estimate the actual load within a short time. When the external load increases suddenly at the 5th second, for example, the vehicle is running through a gravel and sand road. The wheels almost stop under this severe situation. The

Table 2

The specifications of a four-phase motor

\begin{tabular}{llll}
\hline Rated speed & $500 \mathrm{rpm}$ & Maximum speed & $1200 \mathrm{rpm}$ \\
Rated current & $15 \mathrm{~A} / \mathrm{phase}$ & Maximum current & $54 \mathrm{~A} / \mathrm{phase}$ \\
Rated torque & $3 \mathrm{~kg} \mathrm{~m}$ & Maximum torque & $6 \mathrm{~kg} \mathrm{~m}$ \\
Rated power & $1500 \mathrm{~W}$ & Maximum power & $3000 \mathrm{~W}$ \\
\hline
\end{tabular}

driver has to depress the accelerator much more deeply so that the tires exert enough torque to drive the vehicle forward again. It should be noted that slippage is not considered in this paper; the tires are assumed always to adhere fast to the ground.

From Figs. 7 and 8, it is found that the current is distributed to both wheel motors during the 5-13 s interval, according to their different dynamics, but the motors have not obtained enough torque to move the vehicle. After $14 \mathrm{~s}$, the vehicle starts to move, and the total current is controlled to be the same as the driving command. The velocities of both wheels are controlled at the same speed as the vehicle accelerates straight on.

\subsection{Hardware-in-the-loop experiments}

The disk-type axial-flux dc brushless wheel motor is installed on a dynamometer as shown in Fig. 9; its specifications are listed in Table 2. The virtual wheel motor, with a different set of electrical parameters, is coded in a field programmable gate array (FPGA)-Altera Flex-10KE-200SRC240-1, in which the current distribution controller, optimal current command, and PWM generator are also created. The experimental setup is depicted in Fig. 10. The same parameters from Table 1 are used for nominal

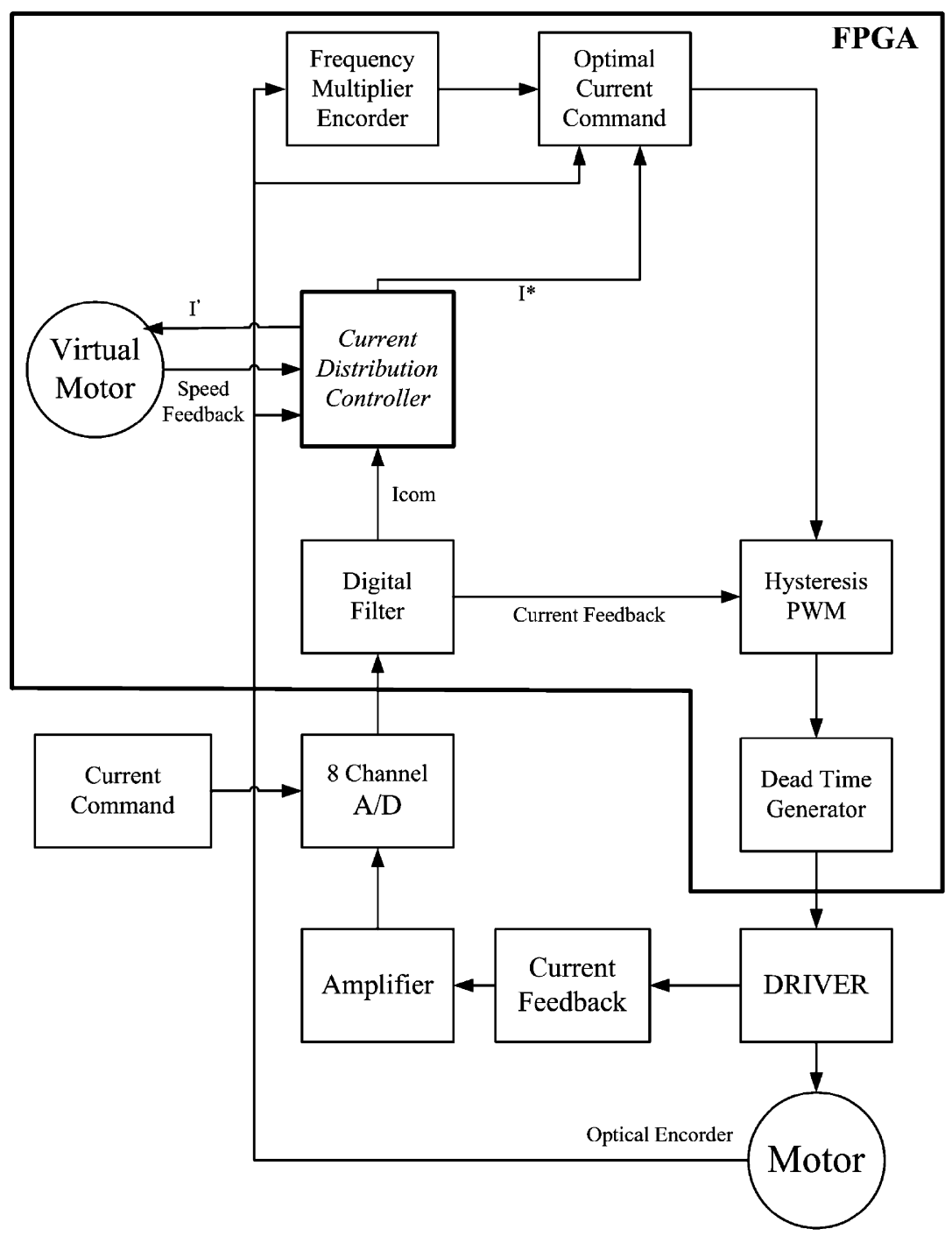

Fig. 10. Experimental configuration. 
values of motor dynamics in the MFC, the PI velocity controllers, and the velocity command compensator.

1. Load disturbance estimation: The external load is generated by the eddy current dynamometer, and is compared with the estimation from the load observer. In Fig. 11, a $0.8 \mathrm{~Hz}$ sinusoidal disturbance is shown on Channel 1 and its estimation from the load observer is on Channel 2. The estimation follows faithfully in phase and magnitude, even for an oscillating signal.

2. Current distribution control: In this experiment, the external load to the virtual wheel motor is fixed at $1 \mathrm{~kg} \mathrm{~m}$. The real wheel motor is subjected to load variation as shown on Channel 4 of Fig. 12. The summation of the current distributed to the real motor on Channel 2 and to the virtual motor on Channel 3 is controlled to be equal to the current command $I_{\text {com. }}$. The current increases on the real motor as the external load is increased, while the current decreases on the virtual one; the current command is successfully distributed by virtue of the VCC and load observer.

3. Velocity control on wheel motors: Under the current distribution control, the velocities of the real and virtual motors are investigated. When the load varies as shown on Channel 4 of Fig. 13, the VCC delivers the velocity command (Channel 1) to adjust current distribution to each motor. The resulting velocity curves of the real and virtual motors are presented on Channels 2 and 3, where two wheels are driven synchronously but with minor inconsistency. This can be resolved by tuning the proportional and integral constants for the velocity controller.

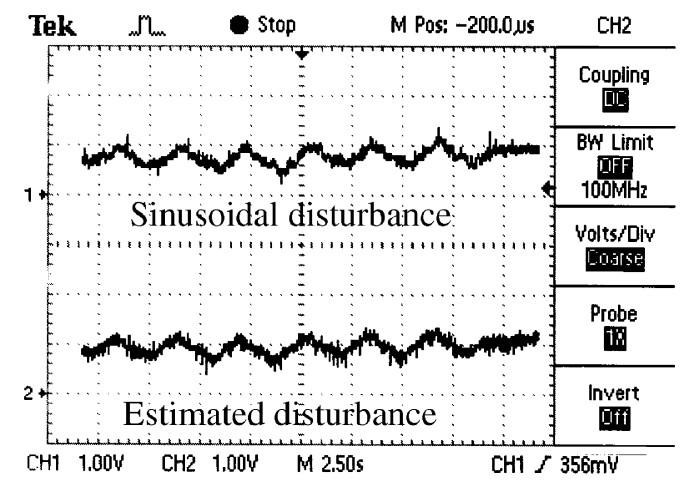

Fig. 11. Estimation of a sinusoidal load disturbance of $0.8 \mathrm{~Hz}(1 \mathrm{~V}: 2 \mathrm{~kg} \mathrm{~m})$.

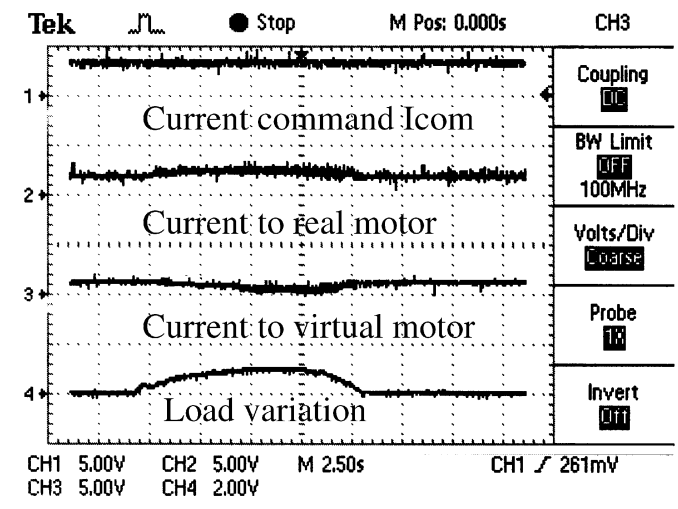

Fig. 12. Current distribution control for a load variation ( $1 \mathrm{~V}$ : $10 \mathrm{~A}$ or $2 \mathrm{~kg} \mathrm{~m}$ ).

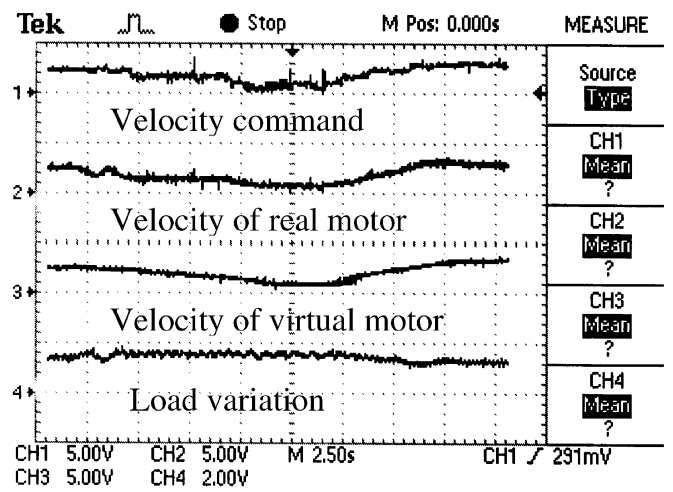

Fig. 13. Velocity control performance under VCC (1 V: $2 \mathrm{~kg} \mathrm{~m}$ or $102.4 \mathrm{rpm}$ ).

\section{Summary and conclusion}

This paper proposes a current distribution control scheme for dual directly driven wheel motors for EVs. This scheme aims to make the driving wheels rotate at a synchronous speed so that the vehicle is kept straight in the cruising mode. Most previous research assumed that all the driving motors on the same vehicle had the same dynamic properties. In other words, the same current input produced the same torque output, or the driving motors had the same torque constant. This is why traction control was developed without considering the differences between driving motors' dynamics. However, this is not true for most cases. Motor dynamics vary due to inconsistent manufacturing quality and unequal aging rate. Under the assumption that there is enough adhesive force between the driving wheels and road surface, slippage does not happen during the proposed current distribution control. This study focuses on the proper distribution of current such that the summation of currents on the driving wheels is the same as the current command from the pedal, and the wheels end up with a synchronous speed.

The current distribution control structure is composed of (i) a pair of model-following controllers for converting the current command into the velocity command through nominal wheel motor models, (ii) a pair of load disturbance observers to estimate external loads from both driving wheels, (iii) a velocity command compensator to generate new velocity commands so that the current command is distributed on the driving wheels without building up excessive energy over the authority of the driver, and (iv) a pair of PI velocity controllers to direct the wheel speeds synchronously in response to the new velocity command.

The conditions of system stability and robustness are analyzed, and verified by simulations of the performance of the current distribution control strategy. Experiments are carried out with a real wheel motor on a dynamometer and a virtual one driven in real time on an FPGA, where the proposed current distribution control strategy is implemented. Experimental data show that the proposed control scheme makes the driving system robust in terms of insensitivity to variation in system parameters and rejection of external disturbances. This current distribution control strategy can be easily extended to the cornering mode by replacing the " $1 / 2$ " blocks in Fig. 3 with specific numbers according to the instantaneous angle of the steering wheel operated by the driver. These numbers can be determined from vehicle geometries, such as the longitudinal length of front and rear wheels and the width of side wheels, and coded into a lookup table on the control chip. The EV research team at the National Taiwan University has been undertaking a project on a hybrid vehicle powered by solar and fuel cells, and the proposed current distribution control strategy will be integrated with advanced 
motion controls, such as slip ratio control, antilock braking system, traction control, and direct yaw control, on the experimental directly driven hybrid EV.

\section{Acknowledgment}

This work was supported by the National Science Council of Taiwan, ROC, under contract NSC 93-2218-E-002-133.

\section{References}

Chen, C. C., \& Chau, K. T. (2001). Modern electric vehicle technology. New York: Oxford University Press.

Hallowell, S.J., Ray, L.R. (2003). All-wheel driving using independent torque control of each wheel. In Proceedings of the American control conference ( $\mathrm{pp}$. 2590-2595), NH, USA: Hanover

Hanselman, D. C. (2003). Brushless permanent-magnet motor design (2nd Ed). New York: McGraw-Hill, Inc.

He, P., Hori, Y., Kamachi, M., Walters, K., Yoshida, H. (2005). Future motion control to be realized by in-wheel motored electric vehicle. The 31st Annual Conference of the IEEE Industrial Electronics Society, IECON 2005, Raliegh, South Carolina, USA.
Hori, Y. (2004). Future vehicle driven by electricity and control-research on fourwheel-motored UOT electric March II. IEEE Transactions on Industrial Electronics, 51(5), 954-962.

Hori, Y., Toyoda, Y., \& Tsuruoka, Y. (1998). Traction control of electric vehicle: basic experimental results using the test EV 'UOT electric march'. IEEE Transactions on Industry Applications, 34(5), 1131-1138.

Mutoh, N., \& Higashikubo, A. (2002). Electric vehicle system independently driving front and rear wheels. In: IEEE 28th annual conference of the industrial electronics society (Vol. 2, pp. 1386-1391). Tokyo, Japan.

Poursamad, A., \& Montazeri, M. (2007). Design of generic-fuzzy control strategy for parallel hybrid electric vehicles. Control Engineering Practice, doi:10.1016/ j.conengprac.2007.10.003.

Pusca, R., Ait-Amirat, Y., Berthon, A., Kauffmann, J.M. (2002). Modeling and simulation of a traction control algorithm for an electric vehicle with four separate wheel drives. In: Proceedings of the IEEE 56th vehicular technology conference (Vol. 3, pp. 1671-1675). Vancouver, Canada.

Sakai, S.-I., Sado, H., \& Hori, Y. (2002). Dynamic driving/breaking force distribution in electric vehicles with independently driven four wheels. Electric Engineering in Japan, 138(1), 761-768.

Yang, Y.-P., Luh, Y.-P., \& Cheung, C.-H. (2004). Design and control of axial-flux brushless DC wheel motors for electric vehicles-part I: Multi-objective optimal design and analysis. IEEE Transactions on Magnetics, 40(4), 1873-1882.

Yang, Y.-P., Wang, J.-P., Wu, S.-W., \& Luh, Y.-P. (2004). Design and control of axialflux brushless DC wheel motors for electric vehicles-part II: Optimal current waveforms and performance test. IEEE Transaction on Magnetics, 40(4), 1883-1891.

Wong, J. Y. (1978). Theory of ground vehicles. New York: Wiley. 